\title{
Experimental Analysis of the Efficiency of Shared Access in IEEE802.15.4-TSCH Networks with Sporadic Traffic
}

\author{
Sahar Ben Yaala \\ Innov'COM Laboratory, Sup'Com \\ University of Carthage, Tunisia \\ National Engineering School of Tunis \\ University of Tunis El Manar \\ email: sahar_by_ing@live.fr
}

\author{
Fabrice Théoleyre \\ ICube Laboratory \\ CNRS / University of Strasbourg, France \\ email: theoleyredunistra.fr
}

\author{
Ridha Bouallegue \\ Innov'COM Laboratory, Sup'Com \\ University of Carthage, Tunisia \\ National Engineering School of Tunis \\ University of Tunis El Manar \\ email: ridha.boualleguedsupcom.tn
}

\begin{abstract}
Industrial wireless networks are now used in many applications, and require to fulfill a certain set of requirements to operate properly. IEEE802.15.4-TSCH is considered a suitable solution to provide real time multihop transmissions in noisy and harsh environments. The standard relies on a strict schedule of the transmissions to reduce the radio duty cycle ratio. While constructing a schedule for periodic traffic has been widely studied in the past, we focus here on the aperiodic, sporadic case. We have to multiplex the transmissions in the schedule to reduce the energy consumption while limiting the number of collisions to provide still high reliability. We propose here to study experimentally the performance of $\mathrm{TSCH}$ with shared access and bursty arrivals. Then, we demonstrate how to readapt the scheduler to better deal with unpredictable traffic. By performing experiments, we can predict the optimal number of transmitters in a shared cell. Thus, we can overcome collisions and packets drops in complex scenarios where bursty traffic is required.
\end{abstract}

Index Terms-IEEE802.15.4-TSCH; sporadic traffic; shared access; ON-OFF traffic; IoT; experimental evaluation

\section{INTRODUCTION}

Low-power wireless networks have been regarded as a key enabler for the Internet of Things. Smart objects such as sensors, mobile devices and actuators are deployed and used for environment sensing and monitoring [1]. In particular, an object measures a physical phenomenon, pushes its measures to a central entity through a wireless infrastructure, and the data is finally stored and processed in the cloud.

The digital Industry 4.0 will comprise a myriad of these devices, able to control and to monitor the manufacturing process [2]. The reconfigurability is now at the heart of the next generation factories: the industrial robots and engines can be reconfigured to change the type of production [3].

The Industrial Internet of Things (IIoT) often needs strong guarantees: the devices have to provide real time statistics with a very high reliability [4]. Because the devices are often battery-powered, the communication stack has to be energyefficient. In particular, a node has to regularly turn off its radio chipset: the lowest the duty cycle ratio, the largest is the lifetime.
IEEE 802.15.4-2015 aims to setup a QoS aware wireless infrastructure for industrial-like wireless networks [5]. TimeSlotted Channel Hoping (TSCH) is among the Medium Access Control (MAC) schemes defined in this standard. TSCH targets specifically the low-power, deterministic and reliable wireless industrial networks. This standard schedules the transmissions such that each application has enough collision-free transmission opportunities. When a node is not involved in a transmission or reception, it can safely turn off its radio interface to save energy.

TSCH is particularly efficient to cope with periodic traffic: some cells can be reserved for each flow, respecting the periodicity. Because different flows have different dedicated cells, no collision arises, and TSCH is able to achieve a very high reliability for constant bit rate (CBR) traffic [6]. Many scheduling algorithms have been proposed to cope with this kind of traffic [7].

However, many industrial applications do not rely on periodic traffic. For instance, event-triggered applications [8] generate a sporadic traffic: the sensor detects an event, and generates a burst of packets, forwarded to the sink. Unfortunately, constructing an accurate schedule to deal with sporadic and aperiodic traffic has received comparatively little attention.

We may use uniquely shared cells for the whole network and their transmissions, using the 6TiSCH minimal configuration [9]. However, as we will highlight it below, such configuration provides a low reliability when the network comprises many nodes. Many contenders may access to the medium simultaneously, generating a large number of collisions.

In this paper, we aim to study more in depth the relevance of using shared cells for the transmission of aperiodic traffic. The contributions of this paper are threefold:

1) we point out the limits of the existing works to construct a schedule based on dedicated cells for an aperiodic traffic with a small delay;

2) we conduct experiments to analyse the reliability obtained by a TSCH network with an aperiodic, ON-OFF traffic model; 
3) we propose guidelines to construct the schedule, grouping some of the devices to use the same group of shared cells.

\section{RELATED WORK}

IEEE802.15.4-TSCH exploits channel hopping and time synchronisation to provide an energy efficient medium access for low-power devices. Because it may rely on a collision-free schedule of the transmissions, it provides high-reliability, and is particularly suitable for the Industrial Internet of Things.

\section{A. Medium Access in TSCH}

In the IEEE802.15.4-TSCH standard, the transmissions are organized within a slotframe repeating over time. The slotframe consists of a matrix of cells, each cell being defined by its timeslot and channel offset. By appropriately scheduling the transmissions in different cells, we can avoid the collisions, which have a very negative impact on both the reliability and the energy efficiency.

Depending on its role in the schedule, a device can decide to transmit, receive or to turn its radio off at the beginning of each slot. The number of elapsed timeslots since the network establishment is computed with an Absolute Sequence Number (ASN) counter. The ASN and the channel offset are then used to derive the physical channel to use at the beginning of each timeslot (eq. 1) [10]:

$$
F=S e q[(A S N+\text { chOffset }) \% \text { seqLength }]
$$

where $F$ denotes the physical frequency to use during the cell, seq[] is a pseudo-random sequence to map an integer to a physical frequency, and seqLength is the length of this sequence. The sequence and the slotframe lengths are mutually prime so that the same cell uses a different physical frequency in consecutive slotframes.

The standard defines two different types of cells:

dedicated cells should be assigned to a group of noninterfering radio links. The transmitter does not implement in that case any contention resolution algorithm since it considers it has a full access.

shared cells are assigned to a group of possibly interfering transmitters. When a transmitter has a packet in its queue at the beginning of a shared cell, it transmits the packet immediately. If a ack is required but wasn't received, the transmitter considers a collision occurred. In that case, it selects a random backoff value, and skips the corresponding number of shared cells.

Let's consider the scenario illustrated in (Fig. 1). In the first attempt, no contention mechanism is activated, but $S 1$ is alone to transmit, and the packet is correctly received. On the contrary, a collision occurs during the next transmission because S1 and S2 have both a frame to transmit. They select a random backoff value, reducing the probability to keep on provoking another collision.

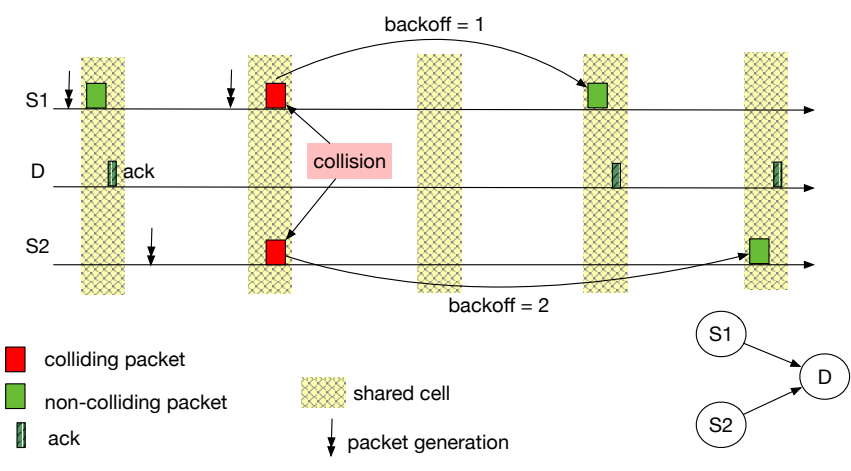

Fig. 1: Transmission within a shared cell.

\section{B. Scheduling the transmissions with TSCH}

Constructing an optimal schedule requires to multiplex the transmissions while avoiding collisions among interfering nodes. Most of the scheduling algorithms focus on a convergecast and periodic traffic pattern, where sensors have to push their measures to the cloud through the border router [7].

When everything is tightly controlled (radio topology, periodic traffic with the instants of generation, etc.), the schedule may be constructed in a centralized manner. Palattella et al. [11] presented a pioneering piece of work to cope with this situation. This algorithm (TASA) relies on matching and coloring techniques to construct a compact schedule, able to minimize the number of timeslots to forward the whole traffic. Additional cells should be provisioned for retransmissions when non perfect links are used to forward the packets. For instance, Yang et al. [12] propose an overprovisionning scheme to handle the link burstiness (i.e. the fact that a link may drop the packets in burst). However, these centralized scheduling algorithms only consider periodic traffic.

The 6TiSCH working group has proposed a simple scheduling function SF0 to insert and remove cells on the fly in the schedule [13]. Each node computes the number of cells required to forward its traffic, using an hysteresis function to avoid oscillations. However, such reactive function was not tailored for an aperiodic traffic, and uses only dedicated cells. Domingo-Prieto et al. also proposed a reactive scheduling approach to handle bursty traffic [14]. However, dedicated cells are reserved even for sporadic traffic, leading to many unused cells.

Duquennoy et al. [15] proposed an innovative way to exploit $\mathrm{TSCH}$, removing the need to negotiate explicitly the cells to use. More precisely, a node uses a pseudo-random sequence to decide which cells to use. However, the number of cells with each neighbor is fixed, whatever the traffic volume is. Elsts et al. have also proposed recently a scheduling algorithm to also address the any-to-any traffic pattern [16]. Each node can receive a certain amount of cells to transmit with each of its neighbors.

We don't have uniquely periodic traffic in the Industrial Internet of Things. In particular, many event-based applica- 
a)
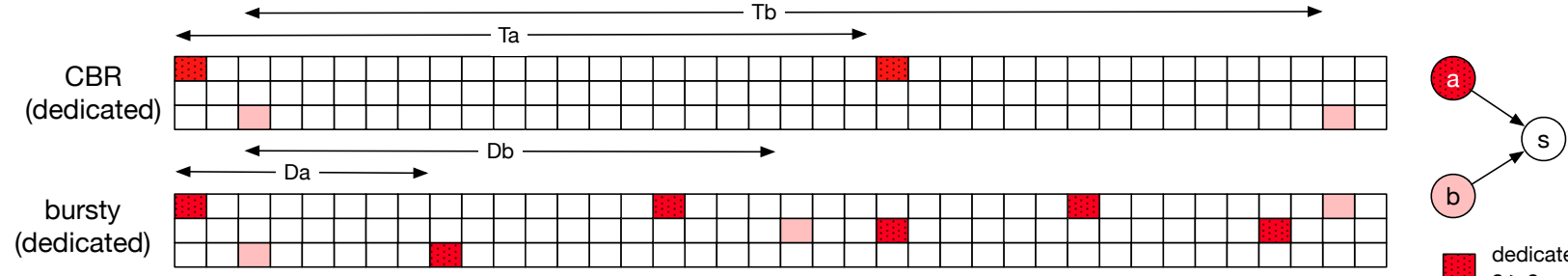

b) (dedicated)

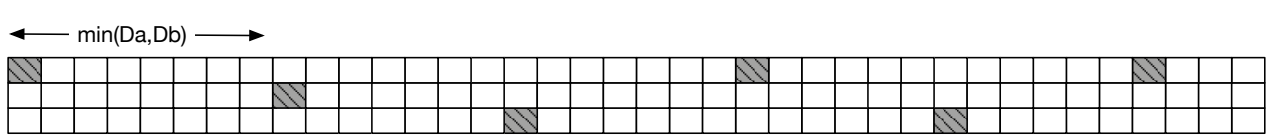

Fig. 2: Possible schedules for periodic, and alarm traffic

tions generate a burst of packets when a physical phenomenon is captured [17]. These flows are delay constrained, and the corresponding packets have to be delivered quickly to the border router. The delay is often appreciated as the first time arrival among the packets of different sensors which all captured the same event.

To the best of our knowledge, scheduling the transmissions for an aperiodic traffic with delay constraints has not yet been addressed in the literature. We aim here to study the relevance of using shared cells to multiplex different flows while reducing the end-to-end delay.

\section{Problem Statement}

Let's consider the schedule for a periodic traffic (case $a$, Fig. 2). We have two transmitters (a and b) which transmit their packets to the sink (s). Since the traffic is periodic, the inter-packet times are fixed ( $T a$ and $T b$ ). We need to schedule accordingly a cell every $T_{a}$ and $T_{b}$ respectively.

Let now consider alarm (sporadic) traffic: the generation of packets is triggered by an event, and is not anymore periodic. However, we may define delay constraints: the alarm has to be delivered to the sink before a given deadline. This deadline may be application specific, possibly non unique for the different nodes. Let denote by $D a$ and $D b$ the deadlines for the flows from $a$ and $b$ respectively. The second schedule in Fig. 2 uses only dedicated cells: it guarantees high reliability since it is collision-free. However, the cells have to respect the deadline constraint: they must be scheduled at least every $D a$ and $D b$ respectively. Because the traffic is sporadic, most of the cells will be probably empty, which impacts the energy consumption.

Let rather consider that shared cells are provisioned in the schedule (case c, Fig. 2). The period of the shared cells have to be sufficiently low to respect the deadline constraint. More precisely, it must take at most the minimum value among the deadline constraints of the different flows. Since the different transmitters may not be active simultaneously, we can multiplex statistically the different flows. Else, a backoff is selected as specified in IEEE8021.5.4-2015 TSCH [5] to regulate the contention. Because this organization uses a small number of cells, the reliability may decrease because of contention. We

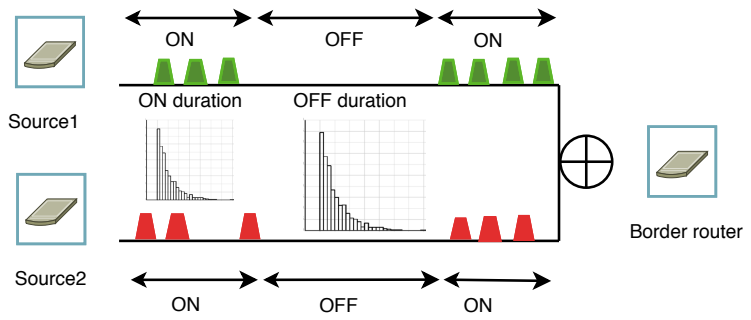

Fig. 3: Proposed topology with exponential ON-OFF arrivals.

aim here to investigate this tradeoff between reliability and efficiency.

We present here an experimental characterization of the usage of shared cells for sporadic traffic with TSCH. We aim to investigate the relevance of using shared cells for alarm (sporadic) traffic. Depending on the obtained measurements, we can decide the maximum number of transmitters that can share the same cell for a given deployment, depending on the traffic characteristics.

\section{Traffic Model and Proposed Scenario}

We consider here a convergecast traffic. We consider that a shared access should be assigned to one receiver, and multiple transmitters (i.e. not all the nodes have to stay awake for each shared cell). In particular, grouping two different receivers in the same shared cells would not bring any benefit: the number of collisions will increase, while using the same amount of energy.

If the cells are unused, only the receivers have to wake up, to listen to the medium and to turn their radio off when no packet is detected after a while.

We consider an aperiodic and sporadic traffic model. Thus, we use an ON-OFF model, where the time between two periods of activity follows an exponential distribution. When a device is $\mathrm{ON}$, it then generates a burst of packets (following a CBR) until it becomes inactive again.

Let's consider the figure 3. During OFF periods, no traffic is generated, while inter $\mathrm{ON}$ durations are picked randomly from an exponential distribution. In order to generate $\mathrm{ON}$ and OFF durations from an exponential distribution with a rate 

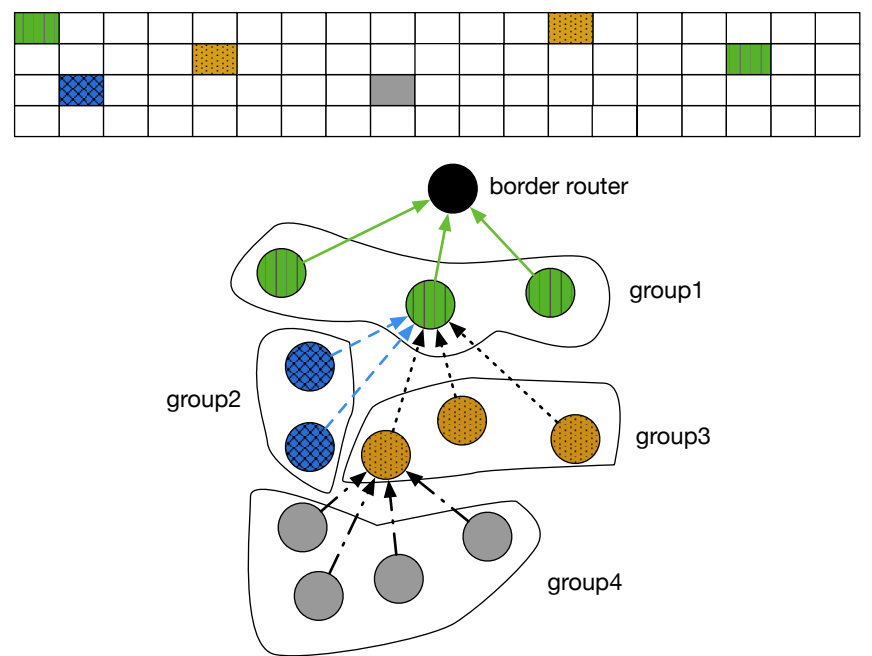

Fig. 4: Scheduling shared cells for different groups of nodes

parameter of $\lambda$, we use a pseudo-random uniform distribution to compute the inter-burst duration as following:

$$
D_{\text {inter-burst }}=-\frac{\ln (\text { random })}{\lambda}
$$

with random denoting the random (uniform) value, picked between 0 and 1 .

To identify the most accurate number of transmitters which should be allocated to a given shared cell, we focus on a star topology, where all the nodes use the same shared cell. We focus on the two following scenarios:

Fixed: a fixed number of shared cells is assigned to a group of nodes. More shared cells means a lower delay, and more opportunities for the retransmissions.

SF0: we modified SF0 to allocate shared (and not anymore dedicated) cells. In particular, the number of shared cells is increased when a node undergoes collisions, so that it has enough bandwidth to retransmit the packets. We tuned SF0 so that the shared cells are removed after a very long timeout, to not remove too aggressively the cells, before a new burst comes.

In multihop, we advocate the usage of a schedule as depicted in Figure 4. The nodes with the same parent are grouped together to share a common cell, so that collisions only arise among siblings. If too much traffic is transmitted inside a group of nodes, it may be split to reduce the volume of collisions. For instance, the groups 2 and 3 have different shared cells although the corresponding devices are attached to the same parent. It is easy to modify the existing distributed or centralized scheduling algorithm to allocate shared cells. In our implementation, a node generates a $6 \mathrm{P}$ request to its parent, which reports the shared cells to use for its children. The parent may decide to assign the node to an existing group (existing shared cells) or to create a new one (new shared cells).

We propose here an experimental study to decide how many

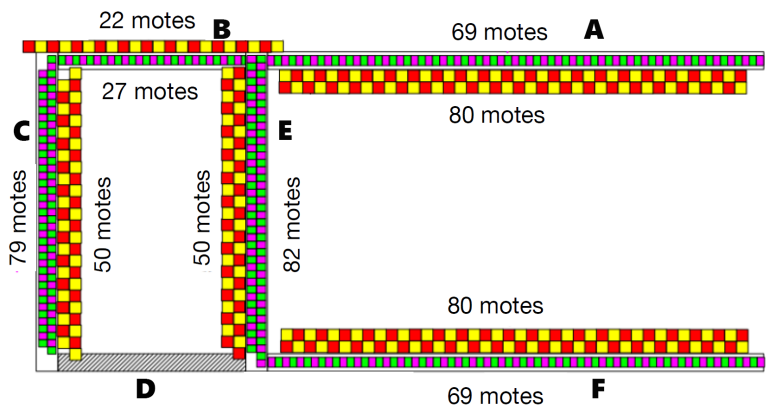

Fig. 5: Grenoble FIT IoT-LAB testbed map.

devices should be assigned at most to the same group of shared cells to respect a given minimum reliability.

\section{EXPERIMENTAL SETUP}

The experimental study was conducted over the fully instrumented testbed FIT/IoT-LAB in Grenoble. It provides the opportunity to evaluate thoroughly any protocol and algorithm for an industrial indoor wireless network. Nodes are located in corridors and spread across an area of $65 \mathrm{~m} * 30 \mathrm{~m}$ (cf. Fig 5).

Experiments are carried out with the M3 nodes. They are based on ARM Cortex-M3 micro-controllers (32-bit) and incorporate AT86RF231, a radio chip compliant with the ISM band.

\section{A. Implementation}

We use here OpenWSN ${ }^{1}$, which provides a full opensource implementation of the IEEE802.15.4-TSCH stack (TSCH, CoAP, etc.) It also integrated the 6TiSCH layers, able to change the schedule on the fly $(6 \mathrm{P})$.

We modified OpenWSN to orchestrate the firmware in order to collect automatically the measures (packets generation, reception, drops). The statistics are forwarded through an out of band interface (the serial line) to not disturb the legal traffic (data and control packets) transmitted via the radio interface. Our modifications are freely available ${ }^{2}$.

At the compilation time, we can choose between:

fixed: the number of shared cells is a priori fixed, common for all the devices. The network is here configured to use either 5 or 10 shared cells;

SF0: we let the SF0 [13] scheduling function decide the number of shared cells to use.

The table I lists all the default values for the different parameters used in our experiments. Our raw measures are publicly available ${ }^{3}$.

\section{B. Performance Criteria}

We measured the following metrics to characterize the network performances:

\footnotetext{
${ }^{1}$ http://www.openwsn.org

${ }^{2}$ https://github.com/benyaalasahar/openwsn-fw/tree/shared

${ }^{3}$ https://github.com/benyaalasahar/dataset-TSCH-sharedCells
} 
TABLE I: Experimental setup.

\begin{tabular}{|c|c|c|}
\hline & Parameter & Default Value \\
\hline \multirow[t]{3}{*}{ Experiment } & Duration & $60 \mathrm{~min}$ \\
\hline & Testbed organization & FIT IoT-Lab Grenoble \\
\hline & Transmission Type & Unicast \\
\hline \multirow[t]{2}{*}{ RPL } & DAO period & $50 \mathrm{~s}$ \\
\hline & DIO period & $8.5 \mathrm{~s}$ \\
\hline \multirow{5}{*}{$\mathrm{TSCH}$} & Slotframe length & 101 \\
\hline & NShared cells & 5 \\
\hline & Timeslot duration & $15 \mathrm{~ms}$ \\
\hline & Maximum retries & \\
\hline & Schedule & $\begin{array}{l}\text { Distributed Random As- } \\
\text { signment }\end{array}$ \\
\hline \multirow[t]{2}{*}{ Queues } & Timeouts & $8 s$ \\
\hline & Queue size & 10 packets \\
\hline \multirow{5}{*}{ Traffic } & $\begin{array}{l}\text { 1nci. data packets } \\
\text { application }\end{array}$ & $\begin{array}{l}\text { at most o data packets } \\
\text { CoAP }\end{array}$ \\
\hline & Payload size & 48 bytes \\
\hline & type & Bursty (Unicast), expo- \\
\hline & Inter-burst duration & (cf.section VI-A) \\
\hline & $\begin{array}{l}\text { inter-packet time (when } \\
\text { active) }\end{array}$ & $1 \mathrm{~s}$ \\
\hline \multirow[t]{7}{*}{ Hardware } & Antenna & Omnidirectional \\
\hline & Radio chipset & AT86RF231 \\
\hline & Node & ST2M32F103REY \\
\hline & Radio propagation & $2.4 \mathrm{GHz}$ \\
\hline & 802.15.4 Channels & 11 to 26 \\
\hline & Modulation model & O-QPSK \\
\hline & Transmission power & $0 \mathrm{dBm}$ \\
\hline
\end{tabular}

Avg. Delay: time between the packet generation and its reception by the border router. Since the network is globally synchronized, we can measure the delay by counting the number of slots, and multiplying it by the timeslot duration:

$$
\operatorname{delay}(p k)=\left(A S N_{r x}(p k)-A S N_{g e n}(p k)\right) * T_{\text {slot }}
$$

with $A S N_{g e n}$ and $A S N_{r x}$ being respectively the ASN when the packet was generated / received, and $T_{\text {slot }}$ the timeslot durantion. We consider the clock drifts to be sufficiently low to be neglected (typically a few hundreds of $\mu s[18])$.

Distribution of the delays: we study the evolution of the delay over time, to visualize the impact of bursts on the performance.

Packet Delivery Ratio (PDR): the ratio of packets received correctly by the border router and packets generated by the different nodes;

Number of (L2) transmissions: to have an estimation of the robustness of the solution, we forbid the nodes to retransmit the packets. More retransmissions impact both the end-to-end reliability, the energy efficiency and the ability to handle a larger volume of traffic.

We plot systematically the $95 \%$ confidence intervals, when considering series of measures.

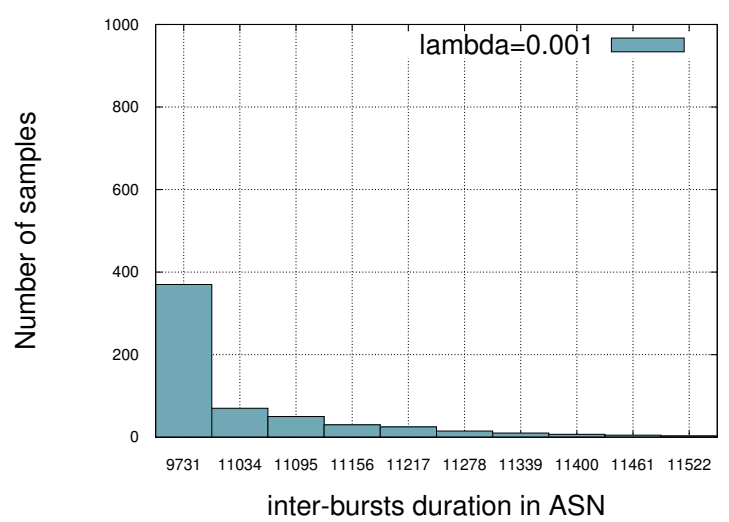

Fig. 6: Inter bursts distribution, lambda $=0.001$.

\section{Shared ACCESS With A VARIABle NUMBER OF TRANSMITTERS (ONE HOP SCENARIO)}

We focus here on a scenario where a fixed number of stations are attached to the same parent, and use a common set of shared cells. We exclude the first seven minutes in each experiment to focus on the steady state, and to not disadvantage SF0 (which needs a few time to converge). Each experiment lasts for one hour to also study the mid-term stability.

\section{A. Validation of the bursty traffic model}

We first here validated our traffic model, generating bursts of packets, with an ON-OFF behavior. The parameter $\lambda$ denotes the traffic intensity and $\frac{1}{\lambda}$ gives the mean parameter of the exponential distribution.

We report in Figure 6 the inter-burst duration. We can verify that we efficiently mimic a bursty traffic, following an exponential distribution.

We consequently consider distinct inter-burst durations depending on the mean parameter of the exponential distribution. The duration of each inter burst $\left(D_{\text {inter-burst }}\right)$ is distributed as follows:

$D_{\text {inter-burst }}$ inASN] $] \in \begin{cases}{[1500,3500],} & \text { if mean }=200 \\ {[3500,5500],} & \text { if mean }=400 \\ {[5500,7500],} & \text { if mean }=600 \\ {[7500,9500],} & \text { if mean }=800 \\ {[9500,11500],} & \text { if mean }=1000\end{cases}$

As illustrated in Figure 6, the duration of each inter-burst varies between 9500 and 11500 (in ASN) for a traffic intensity equal to 0.001 (mean inter-burst duration $=\frac{1}{0.001}$ ).

\section{B. Reliability}

We measured the impact of the number of nodes and the traffic pressure on the performance of the TSCH network (Fig. 7), using both methods to assign the shared cells. We consider a network with 5 (Fig. 7a) and respectively 10 


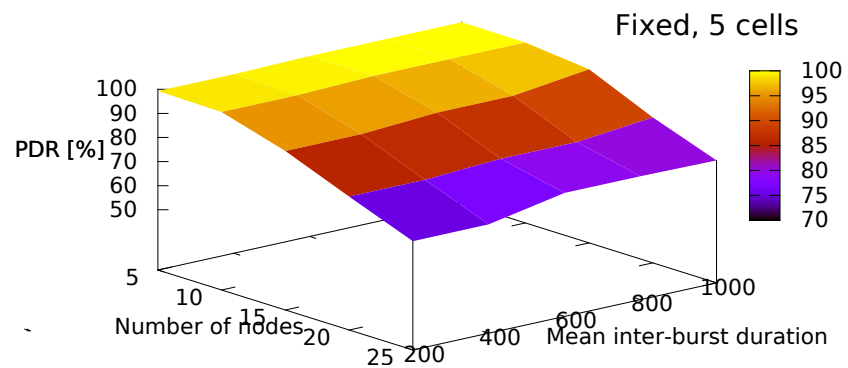

(a) Packet delivery ratio, 5 Fixed cells.

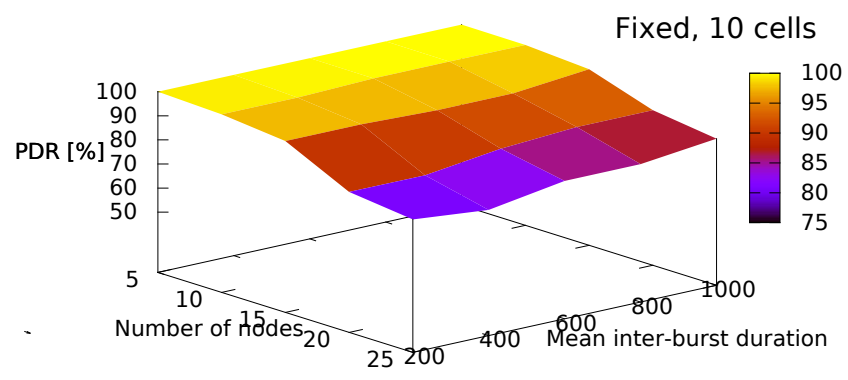

(b) Packet delivery ratio, 10 Fixed cells.

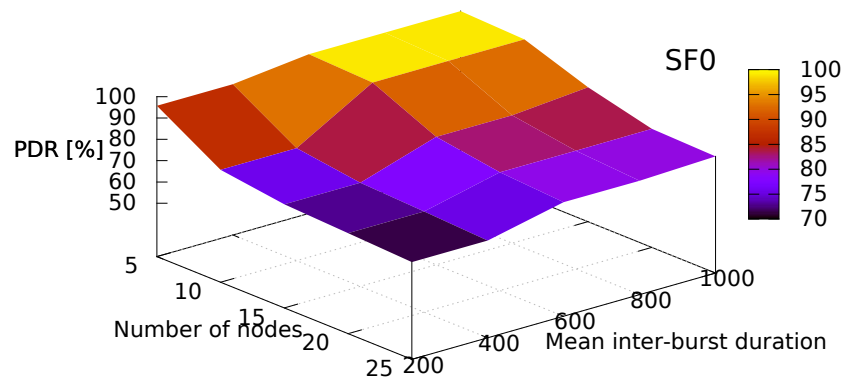

(c) Packet delivery ratio, SF0.

Fig. 7: Impact of the number of nodes and the traffic intensity on the reliability.

(Fig. 7b) shared cells to measure the impact of a static schedule on the reliability.

With a fixed static schedule, all the scheduling approaches achieve to provide very high-reliability with less than 10 nodes, whatever the traffic intensity is. Thus, almost $100 \%$ of the packets are delivered normally to the border router. On the contrary, the reliability begins to drop significantly with more than 15 nodes, even for the smallest traffic intensity $(\lambda=0.001)$. Indeed, more packets mean also more collisions. Since IEEE802.15.4 is very aggressive and drops the packets

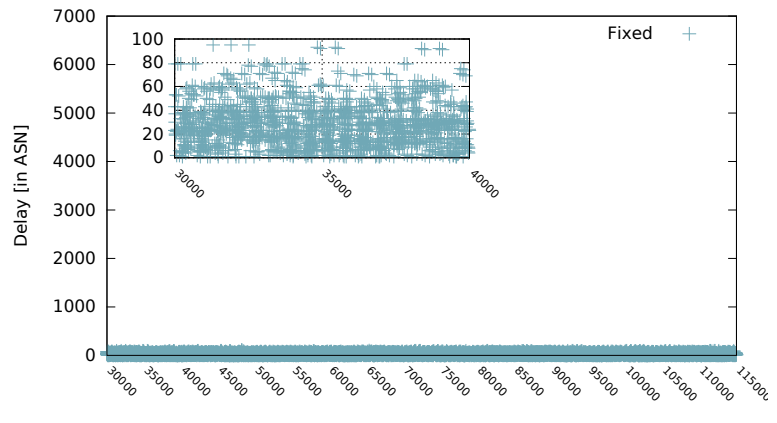

Time (in ASN)

(a) Fixed assignment of 5 shared cells.

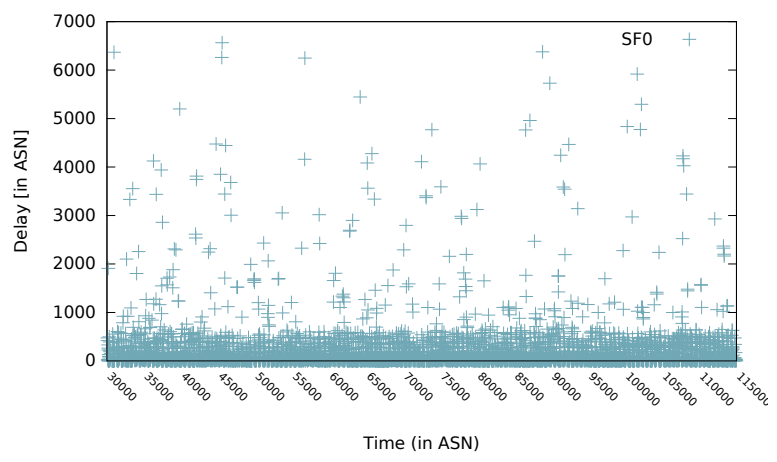

(b) Usage of SF0 to dynamically select the number of shared cells.

Fig. 8: Stability of the delay when using different scheduling schemes, 15 nodes, inter burst duration of $6 \mathrm{~s}$.

after 3 unsuccessful retransmissions by default, the reliability decreases very fast.

With SF0, the cells are reserved on the fly, when traffic has to be forwarded. With very small traffic volumes, SF0 is able to reserve enough cells, and achieves a very highreliability. However, it has been designed rather for a periodic and stable traffic: bursty traffic begins to be dropped because SF0 doesn't succeed to over-provision a sufficient number of cells to handle the storm of collisions. SF0 is not, as expected, the most accurate scheduling function for bursty traffic.

\section{Latency}

We also measured the delay (in number of timeslots) for a small topology of 15 nodes to study specifically the convergence and the stability of the solution (figure 8). With a fixed schedule (Fig. 8a), the shared cells are uniformly distributed. Thus, the delay is very low: the transmitter has just to wait for the next shared cell to transmit its packet. Besides, the delay is very stable all along the experiment, demonstrating the relevance of using shared cells.

On the contrary SF0 reserves cells on the fly. A packet can be generated at any time between two consecutive cells for a given device: the jitter is much larger. Thus, the mean delay is also larger.

Using a fixed number of shared cells allows the network to provide a lower delay and jitter for bursty traffic with even a 


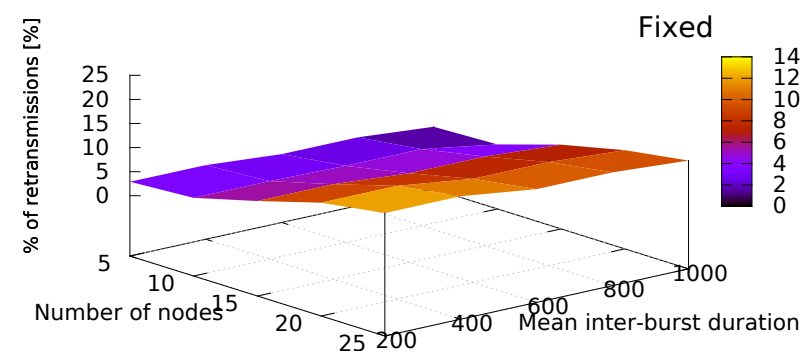

(a) Ratio of retransmissions, 5 fixed cells.

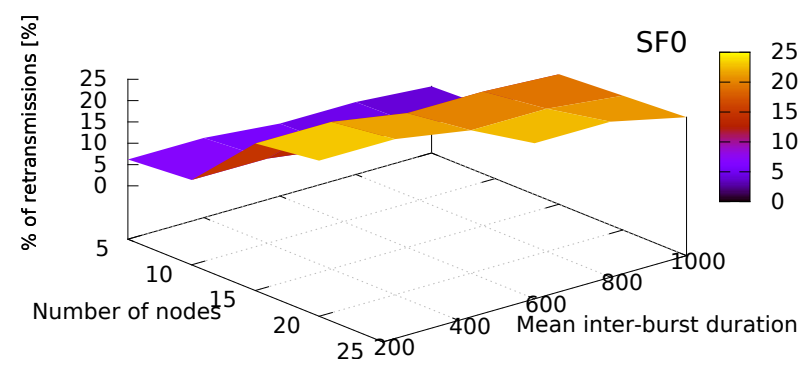

(b) Ratio of retransmissions, SF0.

Fig. 9: Impact of the network size and the traffic intensity on retransmissions.

lower energy consumption for the most loaded nodes.

\section{Efficiency}

Let now focus on the number of transmissions (Figure. 9). It represents a rough estimation of the energy consumption in TSCH. Indeed, a transmitter does not wake-up when it hasn't any packet to transmit, and a receiver can switch-off its radio when it does not sense a packet at the beginning of a timeslot.

With 5 fixed cells, the number of retransmissions increases with the network size (Fig. 9a). More transmitters have to send their packets, and create more collisions. The traffic intensity has only a slight impact because many packets are dropped (the PDR is not equal to $100 \%$ ), and the number of retransmissions has already reached its maximal value (at most 3 retransmissions).

With SF0, the ratio of retransmissions is acceptable only for small network sizes with a small traffic intensity (Fig. 9b). Let us note that the scale is not the same for the graphs for shared cells versus SF0. SF0 has not been designed for bursty traffic, and it tends to under-react to bursts, while releasing and relocating inefficiently the cells.

We finally measured the number of cells reserved in the slotframe. For the shared cells, the number of cells in the schedule is by definition fixed ( 5 or 10 shared cells, depending

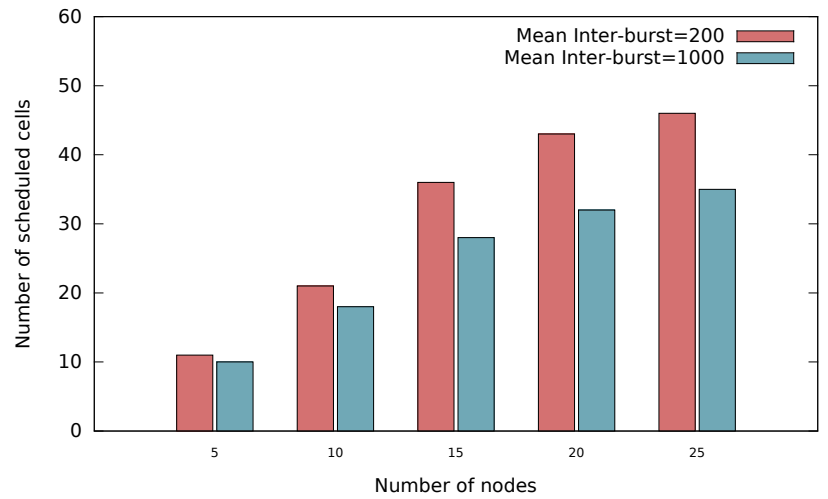

Fig. 10: Impact of the network size and the traffic intensity on the number of cells allocated by SFO.

on the graph). Thus, the figure 10 illustrates the SF0 case. With a larger network, more nodes have to send their packets, and SF0 allocates the cells independently for each source, increasing globally the number of cells reserved by at least one node. Besides, the number of cells is not proportional to the inter-burst duration: SF0 has to provision enough cells, by considering the worst case (i.e. the longest bursts measured so far). 50 cells are reserved for 25 nodes: the probability to have colliding cells with a random assignment is in this case very high [19]. Because some of the cells are unused most of the time, the detection of colliding cells is even trickier. SF0 needs to relocate the allocated cells to solve this unreliability problem.

\section{MUlTiHOP SCENARIO}

We finally considered a multihop scenario. More specifically, we focus on the topology described in Fig. 4. The coordinator is located at the border of the evaluated area, and each radio link connects two nodes 1 meter far away to have good radio links. Each node has a set of shared cells for its children, and different nodes in the DAG have different shared cells (as explained in section IV). The schedule is precomputed, and installed in the device when it boots and has joined the network.

\section{A. Reliability}

We analyzed the impact of the traffic load on the Packet Delivery Ratio (Fig. 11b). The PDR is above 90\%, even for very high traffic loads. This reliability may be sufficient if the different sensors send redundant data. If we need very high reliability ( $P D R \geq 99 \%$ ), we should maintain a sufficiently large inter-burst duration (2,5 minutes between two consecutive bursts).

\section{B. Energy efficiency}

We finally measured the number of retransmissions. Without any surprise, the number of retransmissions decreases with an increasing inter-burst duration (Fig. 11b). Less packets are generated, with less collisions. It is important to notice that 


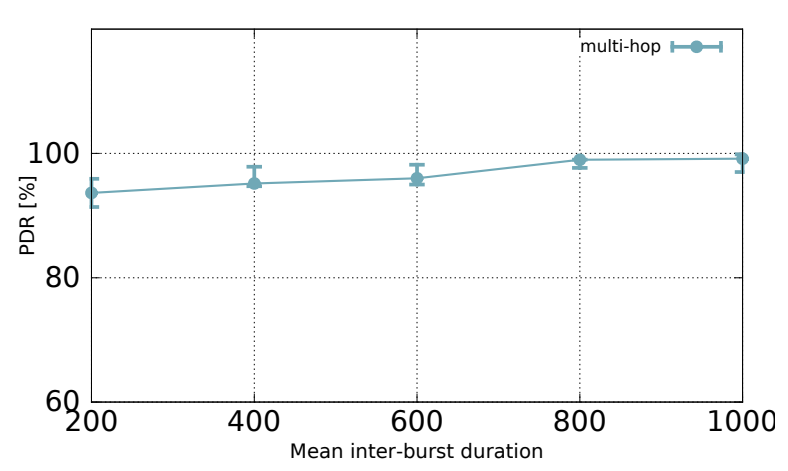

(a) Packet delivery ratio, multi-hop.

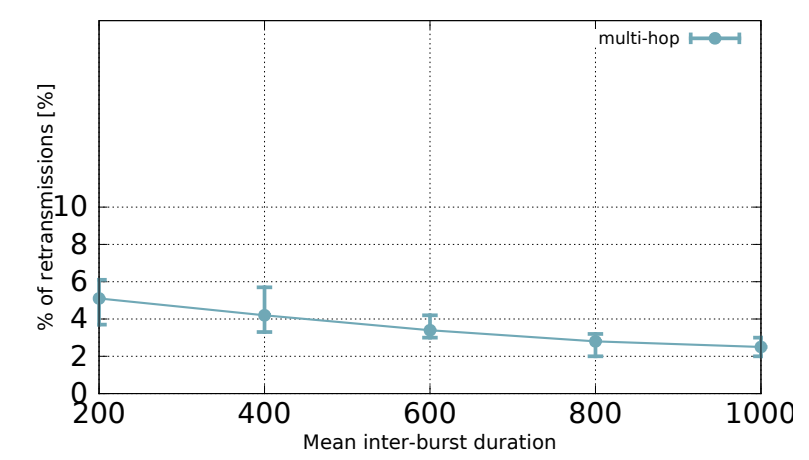

(b) Ratio of retransmissions, multi-hop.

Fig. 11: Impact of the traffic intensity on performance in multihop scenario.

only $6 \%$ of the packets need to be retransmitted even with small inter-burst durations. Thus, shared cells are efficient to handle bursty traffic, even when we may have concomitant bursts.

\section{CONCLUSION}

In this work, we analyzed the capacity of IEEE802.15.4$\mathrm{TSCH}$ to deal with bursty traffic. We thoroughly analyzed its behavior for different exponential ON-OFF arrivals while varying the inter-bursts periods. In particular, we focused on shared access model either with the help of SF0 or through assigning a fixed number of cells. We studied experimentally the amount of collisions and the impact on the reliability for using shared cells to multiplex the different bursts. In this way, we can fix the maximum number of transmitters to allocate to a given shared cell if we aim to guarantee a minimum reliability.

In the future, we aim to propose the algorithms to dynamically tune the number of shared cells in the network In particular, a device should be able to split its children in different groups to minimize the number of collisions. We aim also to investigate the optimal number of groups and the optimal number of shared cells to allocate to minimize the energy consumption.

\section{REFERENCES}

[1] F. Montori, L. Bedogni, and L. Bononi. A collaborative internet of things architecture for smart cities and environmental monitoring. IEEE Internet of Things Journal, 2018.

[2] Martin Wollschlaeger, Thilo Sauter, and Juergen Jasperneite. The future of industrial communication: Automation networks in the era of the internet of things and industry 4.0. IEEE Industrial Electronics Magazine, 11(1):17-27, 2017.

[3] Shiyong Wang, Jiafu Wan, Di Li, and Chunhua Zhang. Implementing smart factory of industrie 4.0: An outlook. Int. J. Distrib. Sen. Netw. 2016:7:7-7:7, January 2016.

[4] Mohammed E El-Telbany and Maha A Maged. A review of wireless sensor applications in remote sensing and space industry. In International Conference on Advanced Intelligent Systems and Informatics, pages 895-904. Springer, 2017.

[5] Std 802.15.4-2015 (Revision of IEEE Std 802.15.4-2011). Low-Rate Wireless Personal Area Networks (LR-WPANs). Technical report, IEEE, April 2016.

[6] Sahar Ben Yaala, Fabrice Théoleyre, and Ridha Bouallegue. Cooperative resynchronization to improve the reliability of colocated ieee 802.15. 4tsch networks in dense deployments. Ad Hoc Networks, 64:112-126, 2017.

[7] Rodrigo Teles Hermeto, Antoine Gallais, and Fabrice Theoleyre Scheduling for ieee802.15.4-tsch and slow channel hopping mac in low power industrial wireless networks: A survey. Computer Communications, 114:84-105, 2017.

[8] N. Nikaein, M. Laner, K. Zhou, P. Svoboda, D. Drajic, M. Popovic, and S. Krco. Simple traffic modeling framework for machine type communication. In International Symposium on Wireless Communication Systems (ISWCS). IEEE, Aug 2013.

[9] X. Vilajosana, K. Pister, and T. Watteyne. Ipv6 over the tsch mode of ieee 802.15.4e. RFC 8180, IETF, May 2017.

[10] Chao-Fang Shih, Ariton E Xhafa, and Jianwei Zhou. Practical frequency hopping sequence design for interference avoidance in $802.15 .4 \mathrm{e}$ TSCH networks. ICC, 2015

[11] M. R. Palattella, N. Accettura, L. A. Grieco, G. Boggia, M. Dohler, and T. Engel. On optimal scheduling in duty-cycled industrial iot applications using ieee802.15.4e tsch. IEEE Sensors Journal, 13(10):36553666 , Oct 2013.

[12] H. T. Yang, K. S. Liu, J. Gao, S. Lin, S. Munir, K. Whitehouse, and J. Stankovic. Reliable stream scheduling with minimum latency for wireless sensor networks. In International Conference on Sensing, Communication, and Networking (SECON), pages 1-9. IEEE, June 2017.

[13] Diego Dujovne, LA Grieco, Maria Rita Palattella, and Nicola Accettura 6tisch 6top scheduling function zero / experimental (sfx). draft draftietf-6tisch-6top-sfx-00, IETF, 2016.

[14] M. Domingo-Prieto, T. Chang, X. Vilajosana, and T. Watteyne. Distributed pid-based scheduling for 6tisch networks. IEEE Communications Letters, 20(5):1006-1009, May 2016.

[15] Simon Duquennoy, Beshr Al Nahas, Olaf Landsiedel, and Thomas Watteyne. Orchestra: Robust mesh networks through autonomously scheduled tsch. In Proceedings of the 13th ACM conference on embedded networked sensor systems, pages 337-350. ACM, 2015.

[16] Atis Elsts, Xenofon Fafoutis, James Pope, George Oikonomou, Robert Piechocki, and Ian. Craddock. Scheduling high-rate unpredictable traffic in ieee 802.15.4 tsch networks. In International Conference on Distributed Computing in Sensor Systems (DCOSS). IEEE, 2017.

[17] C. Perera, C. H. Liu, S. Jayawardena, and M. Chen. A survey on internet of things from industrial market perspective. IEEE Access, 2:1660-1679, 2014.

[18] Tengfei Chang, Thomas Watteyne, Kris Pister, and Qin Wang. Adaptive synchronization in multi-hop tsch networks. Computer Networks, 76:165-176, 2015.

[19] Inès Hosni and Fabrice Théoleyre. Self-healing distributed scheduling for end-to-end delay optimization in multihop wireless networks with 6tisch. Computer Communications, 110:103-119, 2017. 